\title{
The role of syntactic context in word recognition
}

\author{
GEORGE 0. GOODMAN, JAMES L. McCLELLAND, and RAYMOND W. GIBBS, JR. \\ University of California-San Diego, La Jolla, California 92093
}

\begin{abstract}
This study examines the role of syntactic information in word recognition. Subjects made a word-nonword decision regarding a target string that was preceded by a syntactically appropriate word, a semantically related word, or an unrelated word. In Experiment 1, with syntactic and semantic trials assigned to separate blocks, syntactically and semantically appropriate context significantly reduced lexical decision for subsequent target words, compared with unrelated contexts. In Experiment 2, the syntactically and semantically primed trials were either blocked separately or mixed within the same block. Significant syntactic and semantic effects were both observed in the blocked condition, but only the semantic effect was obtained in the mixed condition.
\end{abstract}

It is a well established fact that context facilitates word recognition (e.g., Tulving \& Gold, 1963), and there is a considerable body of work suggesting that some of these effects are due to semantic priming (Fischler \& Goodman, 1978; Meyer, Schvaneveldt, \& Ruddy, 1975). However, there has been little work specifically designed to examine the effects of syntactic context. One relevant experiment was reported by Miller and Isard (1963), in which they showed that both semantic and syntactic information can improve the processing of sentences. In their first experiment, Miller and Isard's subjects listened to stimulus sentences and were told to repeat the sentences to the experimenter. The stimulus sentences were of three types: meaningful, grammatical sentences (e.g., "The academic lecture attracted a limited audience"), grammatically correct sentences that were semantically anomalous (e.g., "The odorless lecture became a filthy audience"), and ungrammatical sentences (e.g., "From hunters house motorists the carry"). Subjects were most accurate with the meaningful, grammatical sentences, but they were more accurate on the grammatical-anomalous sentences than on the ungrammatical sentences. Thus, appropriate syntactic context resulted in better performance even in the absence of meaningful semnatic information.

Given Miller and Isard's (1969) findings, questions remain regarding the nature of the effect of semantic and syntactic information on linguistic processing. First, do semantic and syntactic information affect word recognition or some other aspect of processing required by the task? In the Miller and Isard experiment, con-

This research was supported by NSF Grant BNS76-14830 to James L. McClelland. Thanks are due to Marguerite Moreno for her assistance in data collection and analysis and Jim Hollan for his helpful comments on an earlier version of this paper. Requests for reprints should be sent to James L. McClelland, Department of Psychology, C-009, University of California, San Diego, La Jolla, California 92093. textual information could have had the effect of improving subjects' performance by providing a structure to facilitate memory retention and/or retrieval, with linguistic structure having no effect on the recognition of the words.

Some results relevant to these issues have been provided by studies using the lexical decision task, at least as far as semantic context is concerned. In the lexical decision task, subjects must determine whether a letter string is a word or nonword. Since lexical decision reaction times (RTs) are faster for words preceded by semantically related words than for words preceded by unrelated words (Meyer et al., 1975), it would appear that the actual process of activating (and accessing) a representation of the stimulus word in memory is influenced by the semantic relation between the words.

To our knowledge, no studies have been carried out to determine whether specifically syntactic (as opposed to semantic) effects on word recognition can be obtained, free of potentially confounding memory storage and retrieval effects. While some studies have used wholesentence primes for target words in the lexical decision task (Fischler \& Bloom, 1979; Schuberth \& Eimas, 1977), the sentences used have been meaningful as well as syntactically correct, and so it has not really been possible to examine the role of syntax in priming separately from the role of semantic context. To explore this contribution while minimizing the problems of memory storage and retrieval, we examined the effect of syntactic priming in the lexical decision task in Experiment 1.

Our approach was to use a single prime word (either an article-like word or a pronoun-like word) that strongly predicted the following word would be of a particular syntactic class (noun or verb, respectively) but had little restrictive effect on the possible meaning of the following word. This also minimized the confounding of semantic with syntactic information. Although it is not 
possible to eliminate semantic information totally when considering real language (as we discuss later), our use of two word "phrases" in the lexical decision paradigm allowed us to separate semantic and syntactic information to the degree that this is possible. For comparison purposes, our experiments also included semantic primes.

\section{EXPERIMENT 1}

\section{Method}

Subjects. Thirty-five students at the University of California, San Diego, participated in the experiment either for money or to fulfill a course requirement. The design called for 32 subjects, but 3 were excluded from analysis for failing to follow instructions and 3 additional subjects were run to replace those eliminated.

Design. Two groups of 16 subjects were run. One group received instructions to attend to the relationships between the prime and target stimuli (the "attend" group). The other group was instructed to ignore relationships between the stimuli (the "ignore" group).

Each subject in either group performed the lexical decision task for two blocks of "semantic" stimuli and for two blocks of "syntactic" stimuli. Excluding warm-up items, each block consisted of 80 pairs of stimuli: 40 word-target pairs and 40 nonword-target pairs. Of the 40 word-target pairs in each block, 20 were appropriately primed and 20 were inappropriately primed. Half the subjects received the two syntactic blocks followed by the two semantic blocks, and the remaining subjects were presented the conditions in the reverse order. Order of blocks within conditions was also counterbalanced.

Materials. For the stimuli used in the syntactic condition, we started with two lists of 20 syntactically appropriate pairs of words (see Appendix A). In each of these lists of 20 pairs, 10 pairs began with an article (including possessive pronouns and the word "no") followed by a noun (the target word) and 10 pairs began with a pronoun (or a very general noun, like "boys") followed by a verb that was usually in the past tense to avoid possible ambiguity regarding the syntactic category of the word. In creating the syntactic stimuli, we avoided any noticeable predictive semantic or associative relationship between syntactic primes and targets. From these pairs, we constructed two blocks of 80 trials each. Each block contained the 20 syntactically related pairs from one of the two lists and 20 syntactically unrelated pairs constructed by pairing the prime used for each of the appropriate pairs with the targets used in the other list, so article-like primes were followed by verbs (e.g., "no agreed") and pronoun-like primes were followed by nouns (e.g., "it planet"). The remaining 40 items were word-nonword pairs constructed by pairing each of the primes with two different nonwords, all of which were pronounceable. Thus, 20 different primes were used in each block, each appearing once with a related word target, once with an unrelated word target, and twice with different nonword targets. No target word or nonword was repeated within a block, but the appropriate targets from one block appeared as inappropriate targets in the other. The same 40 nonwords likewise appeared in both blocks.

The semantic lists were constructed in like manner, using the two lists of 20 pairs of semantically related words shown in Appendix B. The pairs were selected from those used by Fischler (1977). Each set of 20 unrelated pairs was constructed by randomly re-pairing the prime words of one list of 20 related pairs with the target words of the other list of related pairs, with the constraint that all the unrelated pairs be both semantically unrelated and syntactically inappropriate. The 40 nonwords used were different from the 40 used in the syntactic blocks. ${ }^{1}$

The four groups of target stimuli (semantic and syntactic, words and nonwords) were matched in length with all items varying from four to seven letters in length. The semantic target words were matched with the syntactic target words in frequency ( 2 to 395 in Kưera \& Francis, 1967, with a mean frequency of 89 in each group).

Procedure. Subjects were run one, two, or three at a time in individual soundproof booths. Each subject sat in front of a Techtronix Model 602 display unit with the forefinger of each hand resting on one of two keys at the base of the display. The keys were marked "WORD" (the left key) and "NON-WORD" (the right key).

Subjects were told that the experiment concerried language processing and that they would be seeing a series of letter strings, one at a time, on the display in front of them. As each string appeared, they responded by pressing the key marked "WORD" if the string was a word or the key marked "NON-WORD" if the stimulus was not a word. The experimenter stressed that the subject should make the response as quickly and as accurately as possible. Subjects then made simple lexical decisions on 40 single-string practice trials. The 20 words and 20 nonwords in these practice trials were not used at any other point in the experiment, and all subjects were given the same practice list. Each practice trial consisted of a fixation dot presented for $200 \mathrm{msec}$, followed by $800 \mathrm{msec}$ of blank, followed by a word or nonword for which the subjects made a lexical decision. This display remained on until all subjects had responded.

Following the practice block, all subjects were told that there would be two strings of letters (one followed in time by another) on each trial for the remainder of the experiment. The experimenter instructed the subjects to look at both strings on each trial, but to make a word-nonword response only for the second string of the trial. As in the practice trials, subjects were told to respond as quickly and as accurately as possible.

Subjects in both conditions were then told that the pairs of strings they would see in the experiment would sometimes be related in some way. Before each semantic block, the nature of "semantic relatedness" was explained and examples of related and unrelated word pairs were given. Likewise, before each syntactic block, appropriate explanation and examples were given to the subjects. Following these instructions, the attempt to manipulate the subjects' intentions was applied, with the attend group being told that they should pay close attention to any relationships in the stimuli because doing so would help them make their word-nonword decisions and the subjects in the ignore group being told to ignore the relationship between stimuli because it would not help them make their word-nonword decisions.

Subjects then performed on a series of four experimental blocks. Ten practice trials (five word target, five nonword target) were added to the beginning of each experimental block (syntactic or semantic prime-target pairs were used before the syntactic and semantic blocks, as appropriate). The words used in these trials did not appear elsewhere in any of the materials. Each trial consisted of a 200 -msec fixation dot, followed by $300 \mathrm{msec}$ of blank, then a $200-\mathrm{msec}$ presentation of the prime word, then $300 \mathrm{msec}$ of blank, and, finally, the test word, which remained on until all subjects responded. Instructions were repeated before each block. The order of presentation of blocks was counterbalanced across subjects, and each group of three subjects received a different random ordering of the trials within each of the experimen tal blocks.

\section{Results}

Preliminary analysis revealed that the effect of instruction condition (attend vs. ignore) was not significant, and there were no reliable interactions of this factor with other factors. 
Table 1

Reaction Time and Error Results in Experiment 1

\begin{tabular}{|c|c|c|c|c|c|}
\hline & \multirow[b]{2}{*}{ Target } & \multicolumn{2}{|c|}{$\begin{array}{c}\text { Reaction } \\
\text { Time }\end{array}$} & \multicolumn{2}{|c|}{$\begin{array}{c}\text { Percent } \\
\text { Error }\end{array}$} \\
\hline & & Syn & Sem & Syn & Sem \\
\hline \multirow{3}{*}{ Word } & Inappropriate & 549 & 527 & 7 & 3 \\
\hline & Appropriate & 530 & 489 & 3 & 1 \\
\hline & Difference & +19 & +38 & & \\
\hline \multicolumn{2}{|c|}{ Nonword } & 660 & 662 & 4 & 6 \\
\hline
\end{tabular}

Note-Reaction times are given in milliseconds. Syn= syntactic; Sem $=$ semantic.

The RT and error results for word-target trials are shown in Table 1. The data from the primed conditions revealed a significant advantage for lexical decision given syntactically appropriate primes when compared with syntactically inappropriate primes $[\mathrm{F}(1,31)=8.01$, $\mathrm{MSe}=721.16, \mathrm{p}<.01$, for subjects; $\mathrm{F}(1,39)=11.34$, $\mathrm{MSe}=1,315.49, \mathrm{p}<.01$, for items $]$. The semantic priming effect was also significant $[F(1,31)=88.40$, MSe $=260.79, p<.01$, for subjects; $F(1,39)=64.42$, $\mathrm{MSe}=988.38, \mathrm{p}<.01$, for items $]$. The semantic priming effect was significantly greater than the syntactic effect $[\mathrm{F}(1,31)=5.27, \mathrm{MSe}=545.11, \mathrm{p}<.05$, for subjects; $F(1,78)=9.81, \mathrm{MSe}=863.73, \mathrm{p}<.01$, for items] .

The RT and error results for nonword-target trials are also shown in Table 1 . In an analysis of variance using the subject error terms, there were no significant effects of attend vs. ignore instructions $[F(1,30)=1.38$, $\mathrm{p}>.10]$, syntactic vs. semantic list $[\mathrm{F}(1,30)=.93$, $p>.10]$, or the interaction of instructions with list $[\mathrm{F}(1,30)=.06, \mathrm{p}>.10]$.

\section{Discussion}

The result of primary interest is the demonstration that prior syntactic information can influence the time course of word recognition. Lexical decision responses for targets preceded by a syntactically appropriate word were an average of $19 \mathrm{msec}$ faster than responses for words preceded by a syntactically inappropriate prime, and, although the effect was small, it was statistically reliable. Our results also indicated a semantic priming effect that was significantly larger than the syntactic effect. Of course, the size of such an effect reflects the strength of the specific primes tested and in no way suggests that semantics are in some way necessarily stronger than syntax.

\section{EXPERIMENT 2}

Experiment 2 was carried out to explore the syntactic priming effect in two ways. First, we wished to determine whether the fact that we had alerted subjects in Experiment 1 to the possible prime-target relations had any influence on the magnitude of the priming effect. To address this, we simply repeated the conditions of
Experiment 1 for a new group of 16 subjects but deleted all mention of the prime-target relationship. Second, we wished to determine the effect of mixing both semantic and syntactic materials in the same block. Therefore, syntactic and semantic trials in Experiment 2 were either blocked (as in Experiment 1) or mixed within the same block.

\section{Method}

Subjects. Thirty-two undergraduate students at the University of California, San Diego, participated in the experiment. They received either money or course credit.

Materials. The same materials were used as in Experiment 1, but to create four mixed blocks of stimuli, the semantic and syntactic blocks were interleaved in the following way. All of the word pairs beginning with 10 of the syntactic primes (five articles and five pronouns) used in one of the syntactic lists were placed in one list with all of the word pairs beginning with 10 of the semantic primes from one of the semantic lists. The remaining pairs from the same syntactic and semantic lists were combined to produce a second mixed list, and the same procedure was followed again in generating the remaining two mixed lists from the second semantic and syntactic lists. The result was that each mixed block contained 20 syntactic word-target trials (10 appropriately primed, 10 inappropriately primed), 20 semantic word trials ( 10 appropriately primed, 10 inappropriately primed), and 40 nonword-target trials (20 taken from the syntactic blocks and 20 taken from the semantic blocks). As in the unmixed lists used in the blocked condition, 20 different prime words were each used four times in a given block.

Procedure. The procedure was identical to that used in Experiment 1, with the exception that subjects were not informed of any relationship between prime and target words. They were told only to look at both the first and second strings of letters presented on each trial and to make a word-nonword response regarding the second string.

One-half of the subjects participated in the mixed condition and one-half participated in the blocked condition. As before, the order of presentation of blocks was counterbalanced across subjects. Each target word appeared once in each pair of blocks, with both members of the pair always presented contiguously and ordered such that the second presentation of a given target occurred after an intervening block (e.g., targets in the first block appeared again in the third block).

\section{Results}

The RT and error results of word trials in both blocked and mixed conditions are shown in Table 2. The blocked condition resulted in both syntactic and semantic priming of about the same magnitude as in Experiment 1 . Both the syntactic and semantic primings were significant in the blocked condition [syntactic: $F(1,15)$ $=6.05, \mathrm{MSe}=426.19, \mathrm{p}<.05$, for subjects; $\mathrm{F}(1,39)=$ 4.10, MSe $=1,398.02, \mathrm{p}<.05$, for items; semantic: $\mathrm{F}(1,15)=27.18, \mathrm{MSe}=440.43, \mathrm{p}<.01$, for subjects; $\mathrm{F}(1,39)=20.29, \mathrm{MSe}=1,563.18, \mathrm{p}<.01$, for items]. In the blocked condition, semantic facilitation was still significantly greater than the corresponding syntactic effect $[F(1,15)=9.57$, MSe $=225.32, p<.01$, for subjects; $F(1,39)=9.81, \mathrm{MSe}=863.73, \mathrm{p}<.01$, for items $]$.

The results were quite different in the mixed condition; specifically, there was no significant effect of syntactic priming $[F(1,15)=.33, \mathrm{MSe}=402.52, \mathrm{p}>.10$, 
Table 2

Reaction Time and Error Results in Experiment 2

\begin{tabular}{|c|c|c|c|c|c|c|c|c|c|}
\hline & & \multicolumn{4}{|c|}{ Reaction Time (in Milliseconds) } & \multicolumn{4}{|c|}{ Percent Errors } \\
\hline & & \multicolumn{2}{|c|}{ Blocked } & \multicolumn{2}{|c|}{ Mixed } & \multicolumn{2}{|c|}{ Blocked } & \multicolumn{2}{|c|}{ Mixed } \\
\hline \multicolumn{2}{|c|}{ Target } & Syntactic & Semantic & Syntactic & Semantic & Syntactic & Semantic & Syntactic & Semantic \\
\hline Word & $\begin{array}{l}\text { Inappropriate } \\
\text { Appropriate } \\
\text { Difference }\end{array}$ & $\begin{array}{l}589 \\
574 \\
+15\end{array}$ & $\begin{array}{r}590 \\
551 \\
+39\end{array}$ & $\begin{array}{r}597 \\
593 \\
+4\end{array}$ & $\begin{array}{l}565 \\
541 \\
+24\end{array}$ & $\begin{array}{l}3 \\
2\end{array}$ & $\begin{array}{l}1 \\
1\end{array}$ & $\begin{array}{l}3 \\
4\end{array}$ & $\begin{array}{l}2 \\
1\end{array}$ \\
\hline Nonword & & 641 & 643 & 650 & 638 & 3 & 4 & 2 & 2 \\
\hline
\end{tabular}

for subjects; $F(1,39)=.15, \mathrm{MSe}=2,081.58, \mathrm{p}>.10$, for items]. The semantic priming effect was still significant $[\mathrm{F}(1,15)=7.47, \mathrm{MSe}=606.52, \mathrm{p}<.05$, for subjects; $F(1,39)=6.38, \quad \mathrm{MSe}=1,687.22, \quad \mathrm{p}<.05$, for items].

The RT and error results for nonwords in both the blocked and mixed conditions are also shown in Table 2. In an analysis of variance treating subjects as the random factor, there were no significant effects of mixed vs. blocked presentation $[F(1,30)=.01, p>.10]$, syntactic vs. semantic list $[F(1,30)=1.20, p>.10]$, or the interaction of blocking condition with list $[F(1,30)=$ $2.10, \mathrm{p}>.10]$.

Analysis of item effects. Examination of the cell means in Tables 1 and 2 reveals that RTs tended to be longer for words in the syntactic condition compared with the semantic condition, even though the words were matched in frequency and length. One possibility is that the differences in results are due to differential effects of syntactic and semantic context, whereas, on the other hand, the difference might be due to uncontrolled differences between the items used in the two lists. To check on the latter possibility, a control group of 16 subjects was run using the same words and nonwords as in the regular experiment, but with all the primes replaced by xs. (It would have been desirable to obtain data for this neutral condition from the subjects who produced data with appropriate and inappropriate primes as well, but pilot testing revealed that mixing these neutral trials with appropriate and inappropriate trials tended to wash out the syntactic and semantic priming effects and did not produce easily interpretable results, as discussed below.)

The results of this control condition revealed that there does appear to be some difference between the target words used in the syntactic and semantic conditions. Mean RT was $547 \mathrm{msec}$ to the words used in the syntactic condition and $526 \mathrm{msec}$ to the words used in the semantic condition, and the difference was statistically reliable over items $[F(1,78)=6.72, \mathrm{MSe}=3,214$, $\mathrm{p}<.01]$.

Further examination of the stimuli used in the syntactic condition revealed that verbs generally produced slower responses than nouns in the syntactic
Table 3

Reaction Times for Noun Primes, Verb Primes, and Semantic Primes

\begin{tabular}{lccc} 
& \multicolumn{3}{c}{ Reaction Time (in Milliseconds) } \\
\multicolumn{1}{c}{ Target } & Verb & Noun & Semantic \\
\hline Neutral & 568 & 530 & 526 \\
Inappropriate & 585 & 547 & 548 \\
Appropriate & 565 & 530 & 510 \\
\hline
\end{tabular}

Note-The results shown for inappropriate and appropriate primes are the combined results of all blocked conditions (i.e., "attend" instructions in Experiment 1, "ignore" instructions in Experiment 1, and blocked condition in Experiment 2).

condition $[\mathrm{F}(1,38)=7.13, \mathrm{MSe}=4,121, \mathrm{p}<.01]$. The noun targets produced RTs quite comparable to those produced by the targets used in the semantic condition (which were also nouns), whereas the verb targets produced longer RTs (see Table 3 ).

To examine these item differences further, the results from the syntactic condition for the three groups of subjects who received the syntactic and semantic materials in separate blocks were further analyzed (these groups include both groups from Experiment 1, plus the blocked condition of Experiment 2). An analysis of variance treating items as the error factor was carried out [items within lexical categories (noun, verb) by priming (appropriate, inappropriate) by group (attend vs. ignore in Experiment 1 vs. blocked in Experiment 2]. The results of interest are shown in Table 3. As with the neutral items, the primed items showed a clear advantage for nouns compared with verbs. The effect of lexical category was significant $[F(1,38)=$ $8.27, \mathrm{p}<.01]$. Inappropriate primes for the nouns produced RTs approximately equal to those produced by inappropriate primes for the semantic words. However, inappropriate primes for verbs produced longer RTs, accounting for the overall difference. The effect of priming was about the same for nouns and verbs, and there was no Priming by Category interaction $[\mathrm{F}(1,38)=$ $.07, \mathrm{p}>.10]$. The group factor did not produce any reliable interactions. The same analysis was attempted on the results from the mixed condition. Although there was a slight trend favoring the nouns over the verbs, it was small and nonsignificant. 


\section{GENERAL DISCUSSION}

Both Experiments 1 and 2 have demonstrated that appropriate syntactic context facilitates word recognition in a lexical decision task. Our experiments also replicate the robust semantic priming effect in recognizing words. The syntactic effect is not large, however, when compared with the semantic priming effect obtained in this and other experiments (e.g., Fischler \& Goodman, 1978; Meyer et al., 1975). It could be eliminated by mixing syntactic and semantic items in the same list, so that the frequency of an appropriate syntactic relation between prime and target was reduced to $25 \%$.

The elimination of a syntactic priming effect (and accompanying reduction of a semantic priming effect) is similar to findings of Tweedy, Lapinski, and Schvaneveldt (1977). In their experiments, Tweedy et al. varied the proportion of primed trials in a lexical decision priming experiment using semantically relevant primes. They found significant priming regardless of the proportion of appropriately primed trials. However, priming was significantly greater when a large proportion of the trials were of the appropriately primed variety ( $87.5 \%$ of all trials) than when a smaller proportion of the trials were appropriately primed (50\%). The priming was smallest (although still significant) when the proportion of appropriate trials was smallest (12.5\% of the trials).

The breakdown of the syntactic priming effect in the mixed condition of Experiment 2 suggests that the use of syntactic information is also at least partially dependent on some appropriate "set" or strategy. It appears that subjects adopt such a set even without specific instructions to do so, or even with specific instructions not to attend to the relation between the prime word and the target.

Recently, Fischler and Bloom (1979) have demonstrated the inability of subjects to eliminate the facilitatory effects of context by ignoring that context. Fischler and Bloom presented subjects with incomplete sentence frames as context, followed by a lexical decision for a target string. Results showed significant priming of the lexical decision by appropriate context. The effect was not changed by instructions to attend to vs. to ignore the context. In Experiment 1 of the present study, we obtained a similar finding, with instructions to attend to vs. to ignore the priming relationships being ineffectual. Jointly, the results of our Experiments 1 and 2 and those of Fischler and Bloom suggest that subjects are either unable or unwilling to avoid priming effects when the priming context bears a reasonably consistent relation to the target.

A number of possible interpretations of the syntactic priming effect might be entertained. First of all, it is possible that the effect is not really priming in the specific sense of preactivating the representation of the target. One possibility is that the syntactic priming effect we observed is one in which the syntactic prime serves to suggest an appropriate morphological analysis of the target word. Perhaps, for example, what a noun prime is doing is suggesting to the subject that the next word should be treated as a verb and thus stripped of a final "-ed" before lexical access. However, if this interpretation were correct, one would expect that the effect of priming would be relatively larger for verbs than for nouns, since the former would require stripping, but not the latter. Such was not the case, as shown in Experiment 2 .

Another possibility is that the observed syntactic priming effect is really a confounding of syntactic and semantic anomaly. Thus, syntactically inappropriate stimulus pairs are also semantically anomalous (e.g., "no-agreed"), which results in a target word's taking longer to recognize. It would be quite useful to totally separate semantic and syntactic information in our experiments. However, in order to do this, it would be necessary to define syntactically inappropriate pairs that were semantically acceptable but not highly semantically related. This appears impossible to do. The difficulty highlights an important point. When using reallanguage stimuli, semantic and syntactic information have some separable and distinct contributions to word recognition and reading, but they are not totally separable. Syntactically appropriate pairs must be semantically acceptable, although they need not provide a large amount of semantic relatedness. Syntactically inappropriate items must also be semantically anomalous, since any semantically acceptable items will also be syntactically acceptable. Therefore, it is important to note that while syntactic information plays a crucial role in word recognition, it does not necessarily prime words totally apart from semantic information.

The idea that syntactic and semantic information are not totally separable is supported by most current models of lexical access and reading. In these models, word recognition occurs primarily from the interaction of both syntactic and semantic information, as well as phonological, orthographic, and pragmatic information (Marslen-Wilson \& Welsh, 1978; Rumelhart, 1977). Nonetheless, our experiments demonstrate that syntactic information does make some contribution to word recognition that is not dependent upon semantic information.

To help us gain further insight into the nature of the mechanism underlying our syntactic priming effect, it would be of great interest to have direct information on whether appropriate syntactic priming facilitates performance or inappropriate syntactic priming inhibits performance. Typically, this question is examined by using a neutral condition, along with appropriate and inappropriate primes (Neely, 1976). We did attempt to include a neutral condition in several unreported condi- 
tions, but we found this unworkable for a variety of reasons. First, as with the list-mixing manipulation, inclusion of neutral primes tended to wash out our priming effects. This "wash out" is puzzling, but predictable, since the inclusion of neutral trials in blocks of appropriately and inappropriately primed lexical decision trials serves to decrease the proportion of appropriate trials. As discussed above, such a reduction in the proportion of appropriate trials has been demonstrated to reduce semantic priming (Tweedy et al., 1977). Second, our preliminary findings suggest that the neutral condition may not have been quite appropriate as a baseline for comparing the effects of a word prime. The problem is that the presentation of a row of $x$ likely has effects on the subject very different from the effects that presentation of a prime word has. A prime word activates a representation of its own meaning, and this activation spreads to the representations of other (related) words, as well. A subject cannot rely exclusively on the activation of the logogen for the target word in making his/her lexical decision responses. Rather, the subject must establish some response criterion that will take such multiple activations into account. However, a row of Xs would probably not activate anything and would leave the subject free to respond whenever the activation of any, single word detector exceeded some criterion.

If the subjects, in fact, adopted a conservative response criterion to cope with multiple activations when the prime was a word, then they would be at a disadvantage in the neutral condition, in which there is no priming word. For these reasons, our experiment does not provide conclusive results on the issue of whether syntactic priming is due to facilitation or inhibition or both. Clearly, it is important to resolve the question of what constitutes an appropriate neutral condition for a given task.

The results of the present study indicate that syntactic information does play a role in processing phrases, even those consisting of only two words. Semantic priming has been demonstrated to involve the operation of two processes: one of automatic spreading activation and a second process that involves conscious processing and reflects a sensitivity to strategies, experimental set, and so on. This type of two-process analysis has also been proposed by Posner and Snyder (1975). On the basis of the current experiment, it seems that syntactic priming is predominantly, if not entirely, due to conscious, strategy-related processes and does not involve an automatic component (at least to the degree of the automatic involvement in semantic priming). It would be possible to further pursue the influence of syntactic information (i.e., devoid of semantic cues) to larger units of text. By varying the amount of syntactically appropriate context preceding a lexical decision, the course of syntactic priming as a function of the amount of constraining syntactic information could be determined.

\section{REFERENCES}

Fischlen, I. Semantic facilitation without association in a lexical decision task. Memory \& Cognition, 1977, 5, 335-339.

Fischler, I., \& Bloom, P. A. Automatic and attentional processes in the effects of sentence contexts on word recognition. Journal of Verbal Learning and Verbal Behavior, 1979, 18, $1-20$.

Fischlen, I., \& Goodman, G. O. Latency of associative activation in memory. Journal of Experimental Psychology: Human Perception and Performance, 1978, 4, 455-470.

KuČera, H., \& Francis, W. N. Computational analysis of present-day American English. Providence, R.I: Brown Universtiy Press, 1967.

Marslen-Wilson, W. D., \& Welsh, A. Processing interactions and lexical access during word recognition in continuous speech. Cognitive Psychology, 1978, 10, 29-63.

Meyer, D. E., Schvaneveldt, R. W., \& Ruddy, M. G. Loci of contextual effects in visual word recognition. In P. M. A. Rabbitt \& A. Dornic (Eds.), Attention and performance $V$. London: Academic Press, 1975.

Miller, G., \& IsArd, S. Some perceptual consequences of linguistic rules. Journal of Verbal Learning and Verbal Behavior, $1963,2,217-228$.

NeELY, J. Semantic priming and retrieval from lexical memory: Evidence for facilitatory and inhibitory processes. Memory \& Cognition, 1976, 4, 648-654.

Posner, M. I., \& Snyder, C. R. R. Attention and cognitive control. In R. Solso (Ed.), Information processing and cognition: The Loyola symposium. Hillsdale, N.J: Erlbaum, 1975.

RUMELHART, D. E. Toward an interactive model of reading. In S. Dornic (Ed.), Attention and performance VI. Hillsdale, N.J: Erlbaum, 1977.

Schuberth, R. E., \& Eimas, P. D. Effects of context on the classification of words and non-words. Journal of Experimental Psychology: Human Perception and Performance, 1977. 3, 21-36.

Tulving, E., \& Gold, C. Stimulus information and contextual information as determinants of tachistoscopic recognition of words. Journal of Experimental Psychology, 1963, 66, 319327.

Tweedy, J. R., Lapinski, R. H., \& Schvaneveldt, R. W. Semantic-context effects on word recognition: Influence of varying the proportion of items presented in an appropriate context. Memory \& Cognition, 1977, 5, 84-89.

\section{NOTE}

1. It should be noted that the syntactic prime words appeared twice as often as the semantic prime words. This was done in order to select syntactic primes that had as little semantic content as possible. Also, we wished to avoid any differential semantic relatedness between the syntactic prime words and their targets. For example, one possible syntactic trial type would be adjective-noun. However, "blue-movie" confounds syntactic and semantic appropriateness more than "blue-bread." For these reasons, we decided to restrict our selection of syntactic prime words to articles and possessive pronouns. 
Appendix A Syntactic Stimulus Pairs

\begin{tabular}{ll}
\multicolumn{2}{c}{ Syntactic Stimulus Pairs } \\
\hline \multicolumn{1}{c}{ List 1 } & \multicolumn{1}{c}{ List 2} \\
\hline whose-planet & your-power \\
their-corner & no-bread \\
our-night & a-rifle \\
no-flower & their-son \\
the-enemy & our-edge \\
her-fund & the-tree \\
my-turkey & any-road \\
your-oven & whose-mud \\
any-kid & my-wife \\
a-thing & her-women \\
you-slid & men-led \\
they-broke & they-sprang \\
people-waved & he-exists \\
men-swear & it-drove \\
it-tied & we-agreed \\
we-paid & you-slept \\
she-kissed & kids-rated \\
he-sent & I-woke \\
I-froze & people-lit \\
kids-play & she-smiled \\
\hline
\end{tabular}

Appendix B Semantic Stimulus Pairs

\begin{tabular}{ll}
\multicolumn{3}{c}{ Semantic Stimulus Pairs } \\
\hline \multicolumn{1}{c}{ List 1 } & \multicolumn{1}{c}{ Appropriate Pairs } \\
\hline door-window & city-town \\
cry-baby & cold-hot \\
bath-clean & short-tall \\
stem-flower & soft-hard \\
anger-mad & high-low \\
king-queen & sell-buy \\
web-spider & lift-carry \\
cut-knife & chair-table \\
fruit-apple & thin-fat \\
sleep-lamb & dog-cat \\
thief-steal & foot-shoe \\
bed-sleep & crowd-people \\
moon-star & salt-pepper \\
hunger-food & black-white \\
rough-smooth & slow-fast \\
dance-sing & sky-blue \\
ocean-water & dark-light \\
gun-shoot & doctor-nurse \\
square-round & thread-needle \\
boy-girl & nail-hammer \\
\hline
\end{tabular}

(Received for publication June 23, 1980; revision accepted March 24, 1981.) 